\title{
Circulating microRNA-762 promotes colorectal cancer proliferation and invasion by upregulating the Wnt-1/ $\beta$-catenin pathway
}

\section{Peng-Sheng Lai}

National Taiwan University Hospital Yun Lin Branch

\section{Wei-Min Chang}

Academia Sinica

\section{Ying-Yin Chen}

National Taiwan University Hospital Yun Lin Branch

Yi-Feng Lin

National Chiayi University

\section{Hui-Fen Liao}

National Chiayi University

Chung-Yu Chen ( $\nabla$ c8101147@ms16.hinet.net )

National Taiwan University Hospital Yun Lin Branch https://orcid.org/0000-0001-9002-7255

\section{Research article}

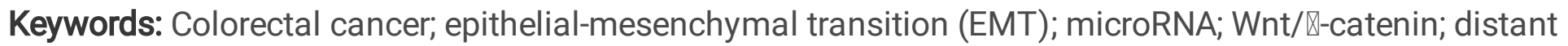
metastasis

Posted Date: November 26th, 2019

DOI: https://doi.org/10.21203/rs.2.17898/v1

License: (9) This work is licensed under a Creative Commons Attribution 4.0 International License. Read Full License 


\section{Abstract}

Background: A number of microRNAs (miRNAs) have been demonstrated to be associated with the diagnosis, progression and prognosis of colorectal cancer (CRC). However, the function of miRNA-762 (miR-762) in CRC remains unclear, and the molecular mechanisms underlying the effects of miR-762 in CRC require further investigation. Methods: The circulating miRNAs from BALB/c mice with CRC CT26 cell implantation were assayed by microarray. Then, miR-762 mimic and inhibitor were transfected to CT26 cells for analysis of cell viability, invasion, and epithelial-mesenchymal transition (EMT), cell cycle, and regulatory molecule expression. Human subjects were included for comparison the circulating miR762 levels in CRC patients and control donors, as well as the patients with and without distant metastasis. Results: The screening for miRNA levels in mice with CRC cell implantation indicated that plasma miR-762 was upregulated. Transfection of miR-762 mimic to CT26 cells increased cell viability, invasion, and EMT, whereas transfection of miR-762 inhibitor decreased the above abilities. Western blot analysis showed that miR-762 mimic transfection upregulated the expression of Wnt-1 and b-catenin, as well as increased the nuclear translocation of b-catenin. Further analysis showed that serum miR-762 levels in CRC patients were higher than in control donors. Among the CRC patients $(n=20)$, six patients with distant metastasis showed higher serum miR-762 levels than the patients without distant metastasis. Conclusions: Circulating miR-762 could promote CRC disease development and progression through the Wnt/b-catenin signaling. miR-762 might be used as a biomarker for CRC diagnosis and targeted therapy.

\section{Background}

Colorectal cancer (CRC) has been ranked among the top five most common cancers worldwide. A number of risk factors have been identified to be involved in the development of CRC, including environmental, lifestyle and genetic factors ${ }^{1}$. The most common treatment for CRC is surgery to remove the tumor before metastasis occurs, which shows a very high cure rate. Moreover, further chemotherapy or target agents such as 5-fluorouracil, camptothecin, bevacizumab, and cetuximab are effective for CRC treatment ${ }^{2}$. However, distant metastasis occurs frequently in most CRC cases resulted in disease progression, and liver and lung metastasis occur in $20-70 \%$ and $10-20 \%$ of patients, respectively ${ }^{3}$. It has been reported that approximately $50 \%$ of patients with CRC develop tumor metastasis which are associated with very poor prognosis and treatment efficiency 4 .

MicroRNAs (miRNAs) are non-translated RNAs that regulate gene expression by degrading mRNA or inhibiting translation ${ }^{5}$. miRNAs have been shown to be involved in many biological processes, such as embryonic development, inflammation, cell cycle regulation, cell differentiation, apoptosis, and tumor metastasis $^{6}$. In recent years, studies have shown that the occurrence of cancer and tumor formation are closely related to miRNA regulation of transcriptional gene expression ${ }^{7}$ and miRNAs can be used as indicators for diagnosing cancer ${ }^{8}$. For example, the miRNAs involved in the development of CRC and 


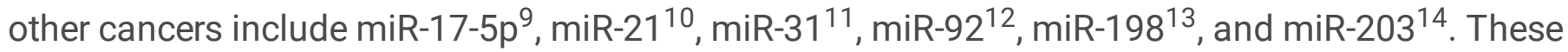
miRNAs can be used as biomarkers for cancer diagnosis or targeted therapy.

Therefore, identifying miRNAs that regulate the growth of CRC and clarifying the regulation of tumor formation and metastasis may reveal disease-specific biomarkers to detect the occurrence or prognosis of cancers. These results would also provide an important foundation for the research and development of advanced treatments in new target therapy strategies. In our initial miRNA array analysis in animal experiments, we detected some miRNAs that may be involved in the growth of CRCs. Among these, plasma miR-762 was found to be upregulated in CRC CT26 cell-transplanted BALB/C mice, suggesting that miR-762 is a potential biomarker for CRC growth regulation. Therefore, we assessed the effects of miR-762 on the viability, colony formation, invasion, cell cycle, and regulatory molecules of CRC CT26 cells by transfection with miRNA mimics and inhibitors. Finally, blood samples were collected from patients with CRC, and the effects of miR-762 in control subjects and cancer cases were compared. These results will provide insight into the role of miR-762 in regulating CRC.

\section{Methods}

\section{Cells and cell culture}

CT26 cells are undifferentiated CRC cell lines produced in N-nitroso-N-methylurethane-induced BALB/C mice. The cells were purchased from American Type Culture Collection (ATCC, Manassas, VA, USA). CT26 cells were cultured in RPMI-1640 medium (Gibco, Grand Island, NY, USA) supplemented with 2 mM Lglutamine and $10 \%$ heat-inactivated fetal bovine serum (FBS; Gibco) at $37^{\circ} \mathrm{C}$, passaged every $2-3$ days with TEG solution ( $0.25 \%$ trypsin, $0.1 \%$ EDTA, and $0.05 \%$ glucose in Hanks' balanced salt solution), and maintained at exponential growth.

\section{Animal experiments and mouse miRNA arrays}

BALB/c male mice (6-8 weeks old), obtained from the National Laboratory Animal Center (Taipei, Taiwan), were maintained in a pathogen-free environment and allowed free access to food and water. All animal experiments were performed according to the guidelines for the care and use of research animals (DHHS publ. NIH 85-23, revised 1996) and the animal use protocol had been reviewed and approved by the institute animal care and use committee (IACUC) of National Yang-Ming University, Taipei, Taiwan (Approval no. 100609).

The mice were divided into two groups: normal control $(n=3)$ and CRC CT26 cell implantation $(n=3)$. As shown in Figure 1A, CT26 cells were prepared as a single-cell suspension in sterile phosphate-buffered saline (PBS) and injected subcutaneously (s.c.) with $2 \times 10^{5}$ cells in the back of mice. Fourteen days after tumor implantation, the tumors had grown to a diameter of $3-5 \mathrm{~mm}$, and then the mice were sacrificed by cervical dislocation euthanasia and blood samples were collected from each mouse. Plasma was 
analyzed by miRNA array (Phalanx Biotech Group, Inc., Hsinchu, Taiwan) according to the manufacturer's protocol. Clustering was performed to visualize the correlations among replicates and under varying sample conditions. Up- and down-regulated genes are represented in red and green colors, respectively. Standard selection criteria to identify differentially expressed miRNAs were established as log2|foldchangel $\geq 0.8$ and $P<0.05$.

\section{Transfection of miR-762 mimic and inhibitor}

CT26 cells were cultured in a petri dish for $4 \mathrm{~h}$ before transfection. miR-762 mimic and inhibitor (miRRibo $^{\mathrm{TM}}$ miRNA, RiboBio Co., Guangzhou, China) were diluted with serum-free RPMI-1640 medium to final concentrations of 50 and $150 \mathrm{nM}$, mixed well, and incubated at room temperature $\left(25 \pm 2{ }^{\circ} \mathrm{C}\right)$ for $5 \mathrm{~min}$. Next, the miR-762 mimic and inhibitor were mixed with Lipofectamine ${ }^{\mathrm{TM}} 2000$ transfection reagent (Invitrogen, Carlsbad, CA, USA), incubated for $20 \mathrm{~min}$, and added to cultured CT26 cells. After $6 \mathrm{~h}$, the medium was removed and fresh RPMl-1640 medium supplemented of 2 mM L-glutamine and 10\% FBS was added for further culture for $24 \mathrm{~h}$.

\section{Cell viability assay}

Cultured CT26 cells were collected and their viability was assayed by the 3-(4,5-dimethylthiazol-2-yl)-2,5diphenyltetrazolium bromide (MTT, Sigma, St. Louis, MO, USA) colorimetric method. Briefly, treated cells were collected, washed with PBS, and reacted with MTT reagent (Sigma) for $2 \mathrm{~h}$ at $37^{\circ} \mathrm{C}$ to allow conversion of tetrazolium salts to a colored formazan product. The optical density of each reaction was measured spectrophotometrically at $550 \mathrm{~nm}$ to calculate cell viability. Cell morphology was observed under an inverted microscope (Olympus, Tokyo, Japan) at a magnification of 200X.

\section{Colony formation assay}

Viable cells $\left(10^{3}\right)$ were plated into $35-\mathrm{mm}$ culture dishes and allowed to grow in RPMI-1640 medium containing $20 \%$ heat-inactivated FBS and $0.3 \%$ agarose at $37^{\circ} \mathrm{C}$ in a humidified $5 \% \mathrm{CO}_{2}$ incubator. After 12 days, the dishes were stained with $0.4 \%$ crystal violet and colony numbers ( 1 colony contained more than 50 cells) were counted.

\section{Cell invasion assay}

Assays of cell invasion properties were performed using a modified Boyden chamber with polyethylene terephthalate filter inserts coated with a Matrigel matrix in 24-well plates containing 8-mm pores. Briefly, 
the cells were suspended in serum-free medium containing $0.5 \%$ bovine serum albumin. These cells were plated into the upper chamber followed by filling the lower chamber with the same medium with or without miR-762 mimic and inhibitor. Cells were incubated for $24 \mathrm{~h}$, and then non-invading cells were gently removed. Cells on the upper side of the filter were carefully removed and cells invading the lower side were counted by microscopic examination (100X, Olympus). For quantification, $10 \%$ acetic acid (100 $\mathrm{mL} /$ well) was used to dissolve stained cells, which were transferred to 96 -well plates for spectrophotometric measurement at $560 \mathrm{~nm}$. For epithelial-mesenchymal transition (EMT) assay, the expression of vimentin and E-cadherin was performed by western blot analysis.

\section{Cell cycle and sub-G1 assay}

The treated CT26 cells were collected, washed with PBS, fixed, and permeated with ice-cold $70 \%$ ethanol overnight, followed by incubation with $0.1 \%$ Triton $X-100,0.2 \mathrm{mg} / \mathrm{mL}$ RNaseA (Sigma) and staining with $20 \mathrm{mg} / \mathrm{mL}$ propidium iodide (PI) for $30 \mathrm{~min}$ at $37^{\circ} \mathrm{C}$ in the dark, and then analyzed by flow cytometry within $1 \mathrm{~h}$. To determine the DNA histogram of cells, data acquisition and analysis were performed on a FACScan flow cytometer with CellQuest software (BD Biosciences, Franklin Lakes, NJ, USA).

\section{Prediction and western blot assay of regulatory pathways}

The targeted genes regulated by miR-762 were predicted by the MIRDB (http://mirdb.org/miRDB/policy.html) and TargetScan systems (http://www.targetscan.org/mmu_61/), while regulatory pathways were analyzed using DAVID Bioinformatics Resources software (NIAID/NIH, http://david.abcc.nciferf.gov/home.jsp). For western blot analysis of predicted molecule expression, the treated CT26 cells were cultured for $24 \mathrm{~h}$, scraped cells from the culture dish, disrupted in $2 \times$ concentrated electrophoresis sample buffer (1 M Tris, pH6.8, 5\% SDS, 40\% glycerol, 0.005\% bromophenol blue, and $8 \%$ b-mercaptoethanol), centrifuged for $5 \mathrm{~min}$ to pellet insoluble material, and subjected to gel electrophoresis on $10 \%$ (wt/vol) SDS-polyacrylamide gels. Protein samples were transferred onto polyvinylidene fluoride (PVDF) membranes. Primary antibodies, including vimentin, E-cadherin, Wnt-1, bcatenin, and MMP-9, were added to the PVDF membrane in TBST (50 mM Tris- $\mathrm{HCl}, \mathrm{pH} 7.4,150 \mathrm{mM}$ $\mathrm{NaCl}, 0.1 \%$ Tween-20) containing $5 \%$ non-fat milk overnight at $4^{\circ} \mathrm{C}$. Subsequently, the PVDF membrane was washed with TBST and incubated with appropriate secondary antibodies (horseradish peroxidaseconjugated goat anti-mouse or anti-rabbit IgG), followed by enhanced chemiluminescence (ECL; Visual protein, Taipei, Taiwan) and the membrane was examined by ECL spectrophotometry (Perkin Elmer, Geliance 600 Camera Imaging system, Waltham, MA, USA). GAPDH was used as an internal control.

\section{Patients and data collection}


The protocols for obtaining clinical samples and information were approved by the Institutional Review Board of National Taiwan University Hospital Yunlin Branch (No. 201611007RINB). Written informed consent to participate in this study was obtained from all of the individuals.

Diagnosis of CRC was based on histological examination of surgical specimens and classified according to TNM staging (Union for International Cancer Control, UICC). The CRC patients are diagnosed for the first time or relapsed, participating in this project before receiving clinical treatment. Patients' basic data were retrieved from electronic medical records and analyzed for age, gender, and tumor staging.

The donors' serum miRNAs were isolated with a TRIZol kit (https://www.ncbi.nlm.nih.gov/pmc/articles/PMC4793896/) and the levels of miR-762 were assessed by

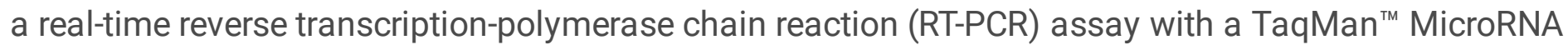
Reverse Transcription Kit (https://www.ncbi.nlm.nih.gov/pmc/articles/PMC2225395/pdf/1746-4811-312.pdf). bdi-miR-159-3p RNA (10 ng/mL serum) was used as a control, and the primers are shown in Table 2.

\section{Statistical analysis}

The results were expressed as the mean \pm standard error (SE) or standard deviation (SD) of at least three experiments. Statistical comparisons were conducted with Student's $t$ test or analysis of variance. A value of $p<0.05$ was considered to indicate a statistically significant difference. All statistical analyses were performed using SigmaStat software (Systat Software, San Jose, CA, USA).

\section{Results}

\section{Expression of miR-762 in BALB/c mice implanted with CRC CT26 cells}

Figure 1A shows the animal experiment flow of BALB/c mice implanted with CRC CT26 cells, and plasma samples from each group were used for miRNA array analysis. Based on the animal experimental design, differentially expressed miRNAs for each comparison were determined by clustering analysis. As shown in Figure 1B, plasma miR-762 levels in CT26 cell-implanted BALB/c mice were higher than those in normal mice according to miRNA array analysis, and the log2 ratio was 2.21 .

\section{Viability and proliferation of CRC CT26 cells transfected with miR-762 mimic and inhibitor}

As shown in Figure 2A, cells transfected with miR-762 mimic showed increased cell viability, whereas those transfected with miR-762 inhibitor showed decreased viability. Transfection with the mimic and inhibitor of miR-762 did change the morphology of CT26 CRC cells (Figure 2B). CT26 cells transfected 
with miR-762 mimic and inhibitor also showed increased and decreased colony formation, respectively, suggesting that miR-762 upregulated cell proliferation (Figure 2C).

\section{Invasion of CRC CT26 cells transfected with miR-762 mimic and inhibitor}

As shown in Figure 3A and 3B, cells transfected with miR-762 mimic showed increased cell invasion ability, while the miR-762 inhibitor decreased cell invasion. This suggests that miR-762 regulates specific genes related to CRC cell invasion. Additionally, miR-762 mimic transfection to CT26 cells increased vimentin and decreased E-cadherin expression, suggesting that miR-762 might upregulate EMT (Figure 3C).

\section{Cell cycle of CRC CT26 cells transfected with miR-762 mimic and inhibitor}

Figure 4A shows that both the miR-762 mimic and inhibitor did not change sub-G1 levels in CT26 cells, suggesting that miR-762 does not regulate cell apoptosis. Moreover, the miR-762 mimic and inhibitor did not cause cell cycle arrest of CT26 cells (Figure 4B).

\section{Signal molecule expression in CT26 cells transfected with miR-762 mimic and inhibitor}

Figure 5A lists the predicted pathways that may be regulated by miR-762. The calcium signaling pathway, endocytosis, MAPK signaling pathway, and Wnt signaling pathway may be related to miR-762 regulation in CT26 cells.

Western blot analysis showed that the miR-762 mimic increased the expression of Wnt-1 and b-catenin, while the miR-762 inhibitor decreased the expression of these molecules (Figure 5B). However, MMP-9 was not changed in either mimic- and inhibitor-transfected cells. Additionally, the cytosolic and nuclear levels of transcription factor b-catenin were both up-regulated in miR-762 mimic-transfected cells (Figure 5C), indicating that miR-762 induced the nuclear translocation of b-catenin. The results suggest that miR762 promotes CRC CT26 cell viability, proliferation and invasion, possibly through the Wnt/b-catenin pathway.

\section{Serum miR-762 level in CRC cancer patients}

The human subjects were enrolled form April to October in 2017. Table 1 shows the basic characteristics of control donors $(n=20)$ and CRC patients $(n=20)$. All of the 20 CRC donors are adenocarcinoma (T2 $T 4, M 0-M 1)$, including colon cancers $(n=14)$ and rectal cancers $(n=6)$. In addition, the present study 
included non-malignant symptom constipation $(n=1)$, colon polyp $(n=6)$, and hemorrhoid $(n=13)$ as control donors.

Table 2 lists the primers used for the RT-PCR assay of serum miR-762 levels. There were no significant differences in gender and age between the two groups. The serum miR-762 level in CRC patients was significant higher than in control donors (Figure 6A). Relative miR-762 expression in CRC patients (T2 $\mathrm{T} 4, \mathrm{M} 0-\mathrm{M} 1)$ was $464.7 \pm 142.3$, while control donors showed a value of $53.2 \pm 11.7(p=0.0073)$. According to the TNM staging, CRC patients with distant metastasis $(M 1, n=6)$ showed significantly higher expression of serum miR-762 than CRC patients without distant metastasis (M0, $n=14)$ (M1 vs. M0, $724.5 \pm 872.3$ v.s. $129.2 \pm 142.1, p=0.019$ ) (Figure 6B). The results demonstrated that miR-762 might promote CRC growth and metastasis.

\section{Discussion}

miRNAs are a group of endogenous, small (18-25-nucleotide long), and non-coding RNA molecules that regulate the expression of specific mRNAs by either translational inhibition or mRNA degradation ${ }^{5}$. More than $50 \%$ of miRNAs are reported to be in cancer-associated genomic break points and function as tumor suppressors or oncogenic molecules ${ }^{6}$. Our study has screened circulating miR-762 expression in mice with CRC CT26 cell implantation. Further investigation demonstrate that CT26 cells transfected with miR762 mimic promoted the cell viability, invasion and EMT of CRC cells. A similar result was also seen in human subjects. Expression of miR-762 in circulation was significantly higher in clinical CRC patients than in control donors. We subsequently confirmed that miR-762 significantly increased in distant metastasis of CRC, resulting in poor prognosis. Our study of the biological roles of miR-762 in CRC revealed Wnt/b-catenin as a downstream signaling pathway. These results in animal model, cell culture, and CRC patients suggested that miR-762 is a crucial factor that stimulates CRC growth and invasion, and such effects may be regulated by the $W n t / \beta$-catenin pathway.

miR-762 is upregulated in radiation-induced tumors in mice and may affect the pathways involved in apoptosis in this context ${ }^{15}$. Several studies reported abnormal miRNA expression in numerous cancers, and miR-762 overexpression has been reported in breast cancer and ovarian cancer, where it promoted cancer cell growth and metastasis ${ }^{16,17}$. A previous study also demonstrated an association between miR762 expression and oral carcinogenesis ${ }^{18}$. However, no studies have examined the expression and function of circulating miR-762 in CRC. In this study, CRC cell line with miR-762 transfection was frequently up-regulated the cell viability and EMT, as well as miR-762 detected in serum extracted from CRC patients. Overexpression of miR-762 enhanced CRC cell proliferation and invasion. The ability of miR-762 to promote cell proliferation and invasion was confirmed by both overexpression and downregulation experiments in vitro. Thus, our results suggest that miR-762 is a novel oncogenic RNA in CRC.

To explore the molecular mechanisms by which miR-762 enhances CRC cell growth and invasion, Wnt/b-catenin was identified as a direct target of miR-762 in CRC cells. First, the Wnt/b-catenin signaling pathway was predicted to be regulated by miR-762 ${ }^{19,20}$. Second, western blot analysis showed that the 
miR-762 mimic increased the expression of Wnt-1 and b-catenin. Third, the cytosolic and nuclear levels of transcription factor b-catenin were both up-regulated in miR-762 mimic-transfected cells. However, CT26 cells transfected with the mimic and inhibitor of miR-762 did not show altered cell cycle or changes in sub-G1 levels in CT26 cells. Additionally, MMP-9 was not changed in either mimic- and inhibitortransfected cells. These data suggest that miR-762 regulates CRC CT26 cell viability, invasion and EMT might through the Wnt/b-catenin pathway, but does not regulate cell cycle and apoptosis.

miR-762 exerted its regulatory effects on cell proliferation, invasion and EMT by affecting the Wnt/ $\beta$ catenin signaling pathway. $\beta$-catenin can influence metastasis through its effects on the $\mathrm{EMT}^{21}$. An article reported that FOXP3 interacts with $\beta$-catenin could induce transcription of Wnt target genes to promote cell proliferation, invasion and EMT induction ${ }^{22}$. Hou et al. reported that miR-762 can downregulate the expression of a tumor suppressor protein Menin through a binding site in its 3'-UTR and consequently upregulate the Wnt cell signaling pathway to promote the development of ovarian cancer ${ }^{17}$. The present study demonstrated a similar result in CRC that miR-762 promoted CRC cell metastasis by promoting the Wnt/ $\beta$-catenin pathway according to the results of the transwell invasion assay and EMT molecules (vimentin and E-cadherin) by Western blotting.

Although miR-762 is overexpressed in several cancers, no studies have investigated the clinical role of miR-762 in CRC. In this study, real-time PCR confirmed that the expression of serum miR-762 in CRC patients was much higher than that in control donors. There was also an association between serum miR-762 expression and CRC patients with distant metastasis. Our study first revealed that circulating miR-762 expression may be useful as a biomarker for diagnosing CRC and higher serum miR-762 expression-caused distant metastasis of patients with CRC. While patients with high expression of miR762 may have shorter survival times than those with low expression of miR-762, survival analysis could not be performed because of the relatively small sample size and short time. Furthermore, the associations between serum miR-762 and tumor size and lymph node invasion were not thoroughly investigated. Therefore, further large-scale studies are necessary to investigate whether serum miR-762 levels can be used as a predictive and a prognostic factor of patients with CRC.

\section{Conclusions}

The present study demonstrated the following: (1) In BALB/c mice, plasma miR-762 levels in CT26 cell-implanted mice were higher than those in normal mice determined by miRNA array analysis. (2) In CRC CT26 cells, transfection of miR-762 mimic increased cell viability, colony formation, cell invasion, EMT, and Wnt/b-catenin expression. (3) In humans, serum miR-762 levels in CRC patients were higher than in control donors according to the RT-PCR assay. (4) Higher serum miR-762 levels in CRC patients induced distant metastasis. Therefore, miR-762 upregulation in CRC may be useful as a biomarker for the clinical diagnosis and prognosis. New drug development of miR-762 inhibitors or blockers may be an effective strategy for CRC treatment. 


\section{Abbreviations}

ATCC: American Type Culture Collection

CO2: carbon dioxide

CRC: colorectal cancer

DHHS: Department of Health and Human Services

DNA: deoxyribonucleic acid

ECL: enhanced chemiluminescence

EDTA: ethylenediaminetetraacetic acid

EMT: epithelial-mesenchymal transition

FBS: fetal bovine serum

GAPDH: glyceraldehyde 3-phosphate dehydrogenase

IACUC: institute animal care and use committee

miR, miRNA: microRNAs

MAPK: mitogen-activated protein kinase

MIRDB: microRNA target prediction database

MMP-9: matrix metallopeptidase 9

MTT: 3-(4,5-Dimethylthiazol-2-yl)-2,5-diphenyltetrazolium bromide

$\mathrm{NIH}$ : National Institutes of Health

PBS: phosphate-buffered saline

PI: propidium iodide

PVDF: polyvinylidene fluoride

RPMI: Roswell Park Memorial Institute

RT-PCR: real-time reverse transcription-polymerase chain reaction

SE: standard error 
SD: standard deviation

SDS: sodium dodecyl sulfate

TBST: Tris buffered saline with Tween-20

UICC: Union for International Cancer Control

\section{Declarations}

\section{Ethics approval and consent to participate}

This study was approved by the Institutional Review Board of National Taiwan University Hospital Yunlin Branch (No. 201611007RINB).

\section{Consent for publication}

Not applicable.

\section{Availability of data and material}

All data generated or analysed during this study are included in this published article.

\section{Competing interests}

No potential conflicts of interest are disclosed.

\section{Funding}

This study was supported by Grants NTUHYL106.C003 from the National Taiwan University Hospital Yunlin Branch for the research and laboratory works.

\section{Authors' contributions}

HFL and CYC conceived and designed the experiments, WMC, YYC and YFL performed the experiments, PSL, HFL and CYC analyzed the data and wrote the paper. All authors have read and approved the manuscript. 


\section{Acknowledgements}

Not applicable.

\section{References}

1. Chang S, Wen S, Chen D, Jin P. Small regulatory RNAs in neurodevelopmental disorders. Hum Mol Genet. 2009; 18(R1): R18-26.

2. Lee JJ, Chu E. Sequencing of antiangiogenic agents in the treatment of metastatic colorectal cancer. Clin Colorectal Cancer. 2014; 13(3): 135-44.

3. Amri R, Bordeianou LG, Sylla P, Berger DL. Variations in metastasis site by primary location in colon cancer. J Gastrointest Surg. 2015; 19(8): 1522-7.

4. Kraus S, Nabiochtchikov I, Shapira S, Arber N. Recent advances in personalized colorectal cancer research. Cancer Lett. 2014; 347(1): 15-21.

5. Wei B, Pei G. microRNAs: critical regulators in Th17 cells and players in diseases. Cell Mol Immunol. 2010; 7(3): 175-81.

6. Bueno MJ, Malumbres M. MicroRNAs and the cell cycle. Biochim Biophys Acta. 2011; 1812(5): 592601.

7. Calin GA, Croce CM. MicroRNA-cancer connection: the beginning of a new tale. Cancer Res. 2006; 66(15): 7390-4.

8. Xie B, Ding Q, Han H, Wu D. miRCancer: a microRNA-cancer association database constructed by text mining on literature. Bioinformatics. 2013; 29(5): 638-44.

9. Ma Y, Zhang P, Wang F, Zhang H, Yang Y, Shi C, Xia Y, Peng J, Liu W, Yang Z, Qin H. Elevated oncofoetal miR-17-5p expression regulates colorectal cancer progression by repressing its target gene P130. Nat Commun. 2012; 3: 1291.

10. Kanaan Z, Rai SN, Eichenberger MR, Roberts H, Keskey B, Pan J, Galandiuk S. Plasma miR-21: a potential diagnostic marker of colorectal cancer. Ann Surg. 2012; 256(3): 544-51.

11. Ng EK, Chong WW, Jin H, Lam EK, Shin VY, Yu J, Poon TC, Ng SS, Sung JJ. Differential expression of microRNAs in plasma of patients with colorectal cancer: a potential marker for colorectal cancer screening. Gut. 2009; 58(10): 1375-81.

12. Motoyama K, Inoue H, Takatsuno Y, Tanaka F, Mimori K, Uetake H, Sugihara K, Mori M. Over- and under-expressed microRNAs in human colorectal cancer. Int J Oncol. 2009; 34(4): 1069-75.

13. Wang J, Du Y, Liu X, Cho WC, Yang Y. MicroRNAs as regulator of signaling networks in metastatic colon cancer. Biomed Res Int. 2015; 2015: 823620.

14. Zhou Y, Wan G, Spizzo R, Ivan C, Mathur R, Hu X, Ye X, Lu J, Fan F, Xia L, Calin GA, Ellis LM4, Lu X. miR-203 induces oxaliplatin resistance in colorectal cancer cells by negatively regulating ATM kinase. Mol Oncol. 2014; 8(1): 83-92. 
15. Gao F, Chen S, Sun M, et al. MiR-467a is upregulated in radiation-induced mouse thymic lymphomas and regulates apoptosis by targeting Fas and Bax. Int J Biol Sci. 2015; 11(1): 109-21.

16. Li Y, Huang R, Wang $L$, et al. microRNA-762 promotes breast cancer cell proliferation and invasion by targeting IRF7 expression. Cell Prolif. 2015; 48(6): 643-9.

17. Hou R, Yang Z, Wang S, Chu D, Liu Q, Liu J, Jiang L. miR-762 can negatively regulate menin in ovarian cancer. Onco Targets Ther. 2017; 10: 2127-37.

18. Yu T, Wang XY, Gong RG, et al. The expression profile of microRNAs in a model of 7,12-dimethylbenz[a]anthrance-induced oral carcinogenesis in Syrian hamster. J Exp Clin Cancer Res. 2009; 28:64.

19. Wu T, Hua X. Menin represses tumorigenesis via repressing cell proliferation. Am J Cancer Res. 2011; 1(6): 726-39.

20. Cao Y, Liu R, Jiang X, et al. Nuclear-cytoplasmic shuttling of menin regulates nuclear translocation of \{beta\}-catenin. Mol Cell Biol. 2009; 29(20): 5477-87.

21. Balasubramaniam SL, Gopalakrishnapillai A, Petrelli NJ, Barwe SP. Knockdown of sodium-calcium exchanger 1 induces epithelial-to-mesenchymal transition in kidney epithelial cells. J Biol Chem. 2017;292(27): 11388-99.

22. Yang S, Liu Y, Li MY, Ng CSH, Yang SL, Wang S, Zou C, Dong Y, Du J, Long X, Liu LZ, Wan IYP, Mok T, Underwood MJ, Chen GG. FOXP3 promotes tumor growth and metastasis by activating Wnt/ $\beta$ catenin signaling pathway and EMT in non-small cell lung cancer. Mol Cancer. 2017;16(1): 124.

\section{Tables}

Table 1. Characteristics of control donors and colorectal cancer (CRC) patients 


\begin{tabular}{|c|c|c|c|c|c|}
\hline \multirow{2}{*}{$\begin{array}{l}\text { Characters } \\
\text { Gender }\end{array}$} & & \multicolumn{2}{|c|}{ Control $(n=20)$} & \multicolumn{2}{|c|}{ CRC $(n=20)$} \\
\hline & & Female: 6 & Male: 14 & Female: 6 & Male: 14 \\
\hline \multirow[t]{2}{*}{ Age (year) } & Mean \pm SE & $63.5 \pm 2.0$ & $67.0 \pm 2.7$ & $57.2 \pm 4.4$ & $64.4 \pm 4.3$ \\
\hline & Range & $57-69$ & $50-87$ & $39-70$ & $41-88$ \\
\hline \multirow[t]{10}{*}{ Diagnosis } & & \multicolumn{2}{|c|}{ Constipation: } & \multicolumn{2}{|c|}{ Colon cancer: } \\
\hline & & \multirow[t]{2}{*}{0} & \multirow[t]{2}{*}{1} & \multicolumn{2}{|c|}{ T2-T3/MO: } \\
\hline & & & & 3 & 6 \\
\hline & & \multicolumn{2}{|c|}{ Colon polyp: } & \multicolumn{2}{|c|}{ T3-T4/M1: } \\
\hline & & \multirow[t]{2}{*}{0} & \multirow[t]{2}{*}{6} & 0 & 5 \\
\hline & & & & \multicolumn{2}{|c|}{ Rectal cancer: } \\
\hline & & \multicolumn{2}{|c|}{ Hemorrhoid: } & \multicolumn{2}{|c|}{ T2-T3/MO: } \\
\hline & & \multirow[t]{3}{*}{6} & \multirow[t]{3}{*}{7} & 3 & 2 \\
\hline & & & & \multicolumn{2}{|c|}{ T3-T4/M1: } \\
\hline & & & & 0 & 1 \\
\hline
\end{tabular}

Table 2. Primers used in this study

\begin{tabular}{ll}
\hline Primers & Sequences \\
\hline Universal miRNA-Reverse primer & GTGCAGGGTCCGAGGT \\
has-miR-762-RT & GTTGGCTCTGGTGCAGGGTCCGAGGTATTCGCACCAGAGCCAACGCTCGG \\
has-miR-762-F & ATTATGGGGCTGGGGCCGGG \\
bdi-miR159-5p-RT & GTTGGCTCTGGTGCAGGGTCCGAGGTATTCGCACCAGAGCCAACGATTGG \\
bdi-miR159-5p-F & GCGGCGAGCTCCCTTCGAT \\
\hline
\end{tabular}

\section{Figures}


(A)

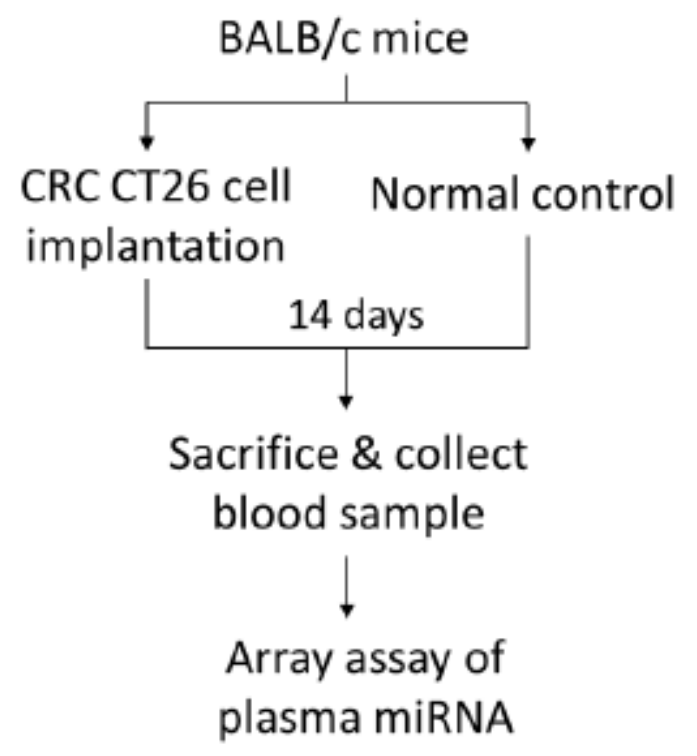

(B)

\section{CRC Control}

$\begin{array}{llllll}1 & 2 & 3 & 1 & 2 & 3\end{array}$

Figure 1

Array assay of plasma miRNA in BALB/c mice with CT26 cell implantation. (A) Animal experiment process, (B) Plasma miR-762 expression by miRNA array. Clustering was performed to visualize the correlations among the replicates and varying sample conditions. Up- and down-regulated genes are presented in red and green colors, respectively. 
(A)

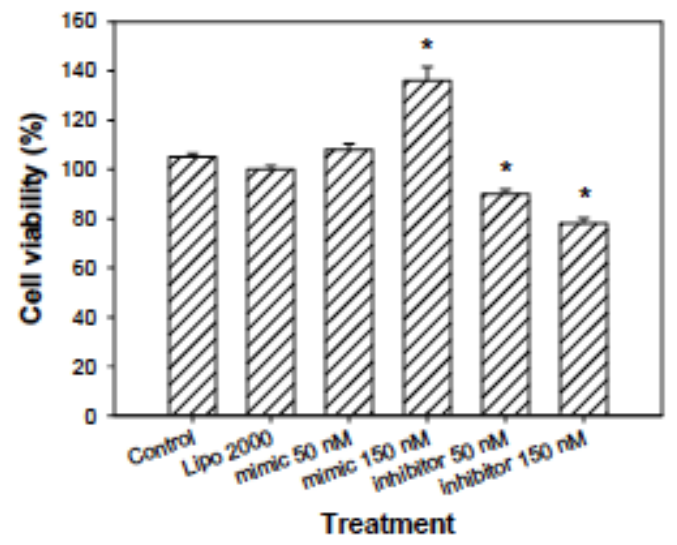

(B)

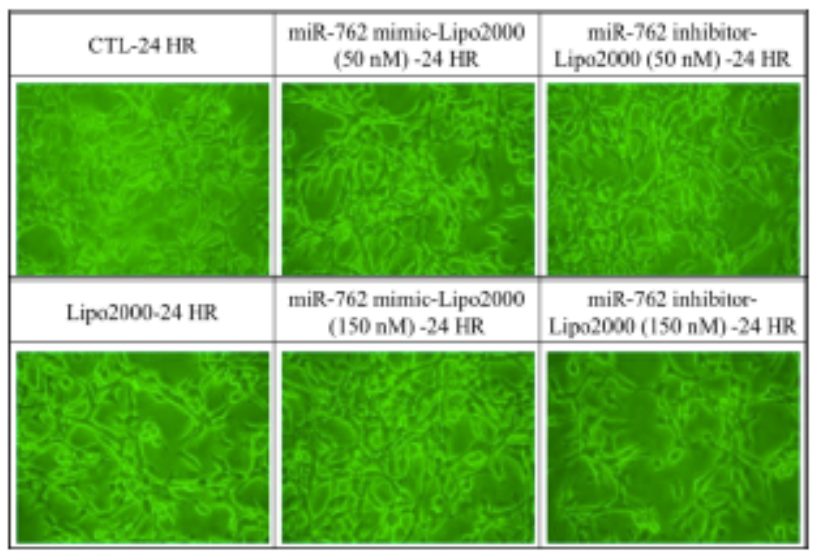

(C)

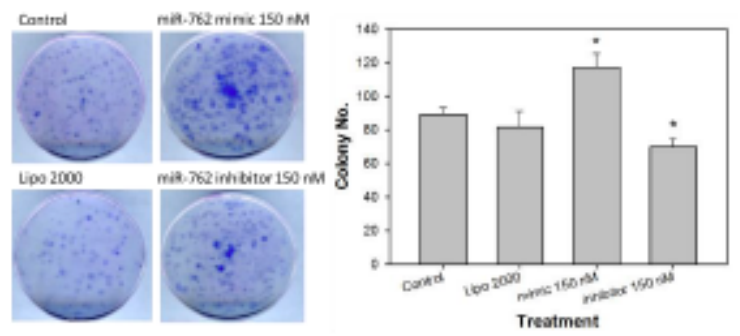

Figure 2

(A) Viability assay, (B) Morphological observation (200X), (C) Colony assay of CT26 cells transfected with mimic and inhibitor of miR-762. Cells transfected with miR-762 mimic $(150 \mathrm{nM})$ increased cell viability and colony number, while miR-762 inhibitor ( 50 and $150 \mathrm{nM}$ ) decreased viability and colony formation by MTT assay. CT26 cells transfected with mimic and inhibitor of miR-762 did not show altered morphology under an inverted microscope view. The results were expressed as the mean \pm standard error (SE) for three independent experiments. Statistical comparisons were conducted with Student's t-test.* $P<0.05$. 
(A)

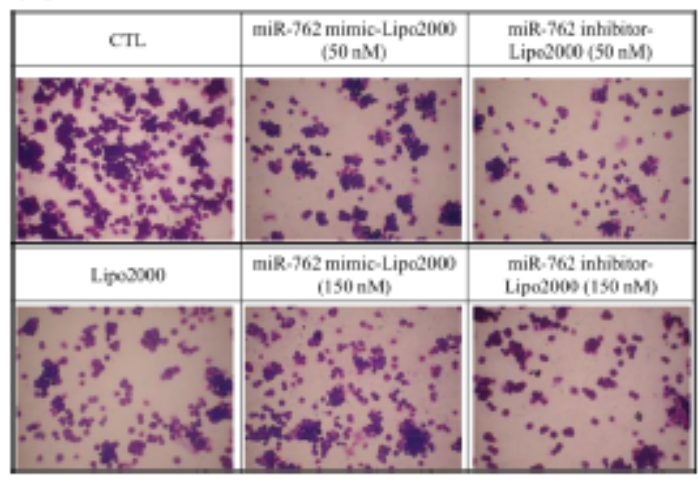

(B)

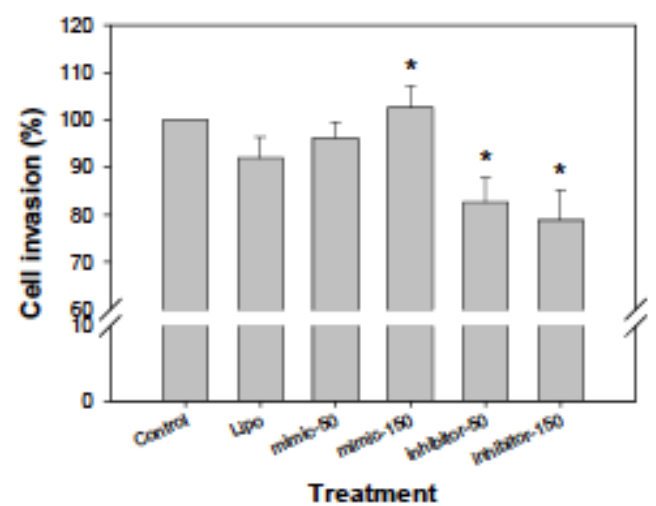

(C)

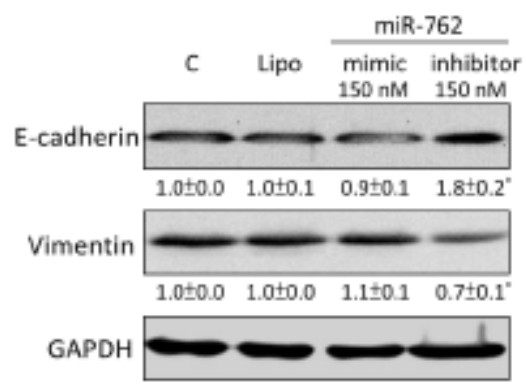

\section{Figure 3}

Invasion assay of CT26 cells transfected with mimic and inhibitor of miR-762. (A) Morphological observation of treated cells (100X), (B) Percent of invasive cells, (C) Expression of vimentin and Ecadherin by western blot analysis. Cells transfected with miR-762 mimic (150 nM) showed increased cell invasion ability, while miR-762 inhibitor (50 and $150 \mathrm{nM}$ ) decreased cell invasion. The results were expressed as the mean \pm standard error (SE) for three independent experiments. Statistical comparisons were conducted with Student's t-test. * $\mathrm{P}<0.05$. 
(A)
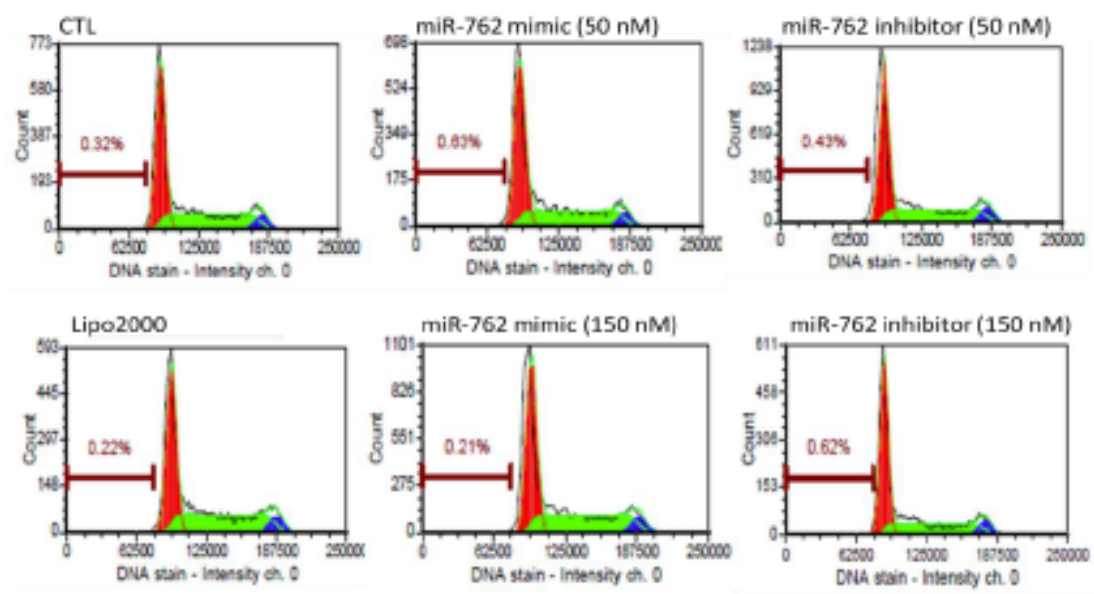

(B)

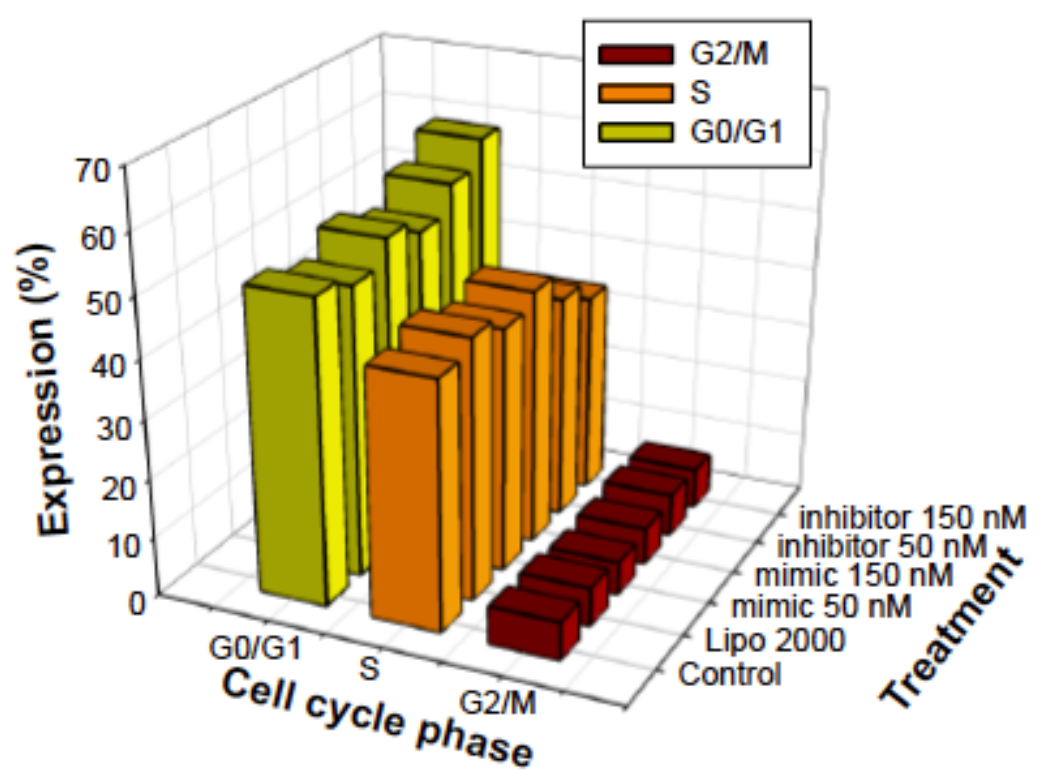

Figure 4

(A) Apoptosis sub-G1, (B) Cell cycle assay of CT26 cells transfected with mimic and inhibitor of miR-762. Neither miR-762 mimic nor inhibitor changed the sub-G1 levels and cell cycle arrest of CT26 analyzed by a FACScan flow cytometer. The results were expressed as the mean \pm standard error (SE) for three independent experiments. Statistical comparisons were conducted with Student's t-test. 
(A)

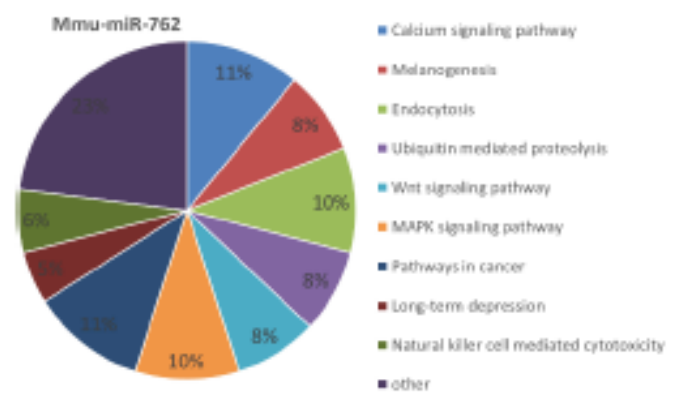

(B)

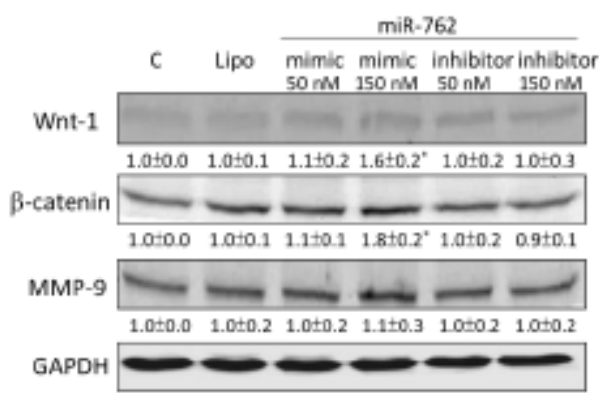

(C)
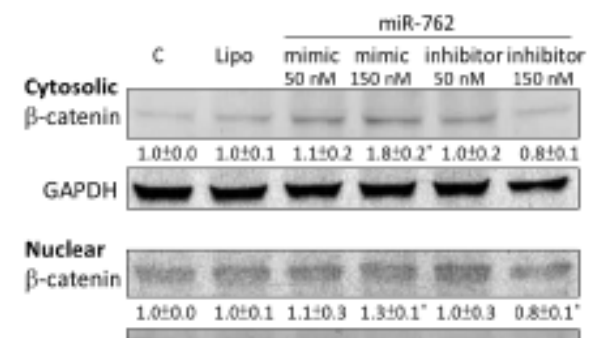

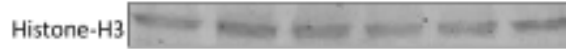

\section{Figure 5}

Wnt/囚-catenin assay of CT26 cells transfected with mimic and inhibitor of miR-762. (A) Prediction of pathways of miR-762 assayed by DAVID Bioinformatics Resources software (NIAID/NIH), (B) Expression of Wnt-1, \-catenin, and MMP-9 by western blotting, and (C) Nuclear and cytosolic levels of $\bigotimes$-catenin. miR762 mimic increased the expression of Wnt-1 and $₫$-catenin, while miR-762 inhibitor decreased the levels of these molecules. MMP-9 was not changed in either mimic- or inhibitor-transfected cells. The cytosolic

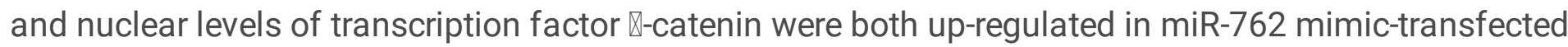


cells. The results were expressed as the mean \pm standard error (SE) for three independent experiments. Statistical comparisons were conducted with Student's t-test. * $\mathrm{P}<0.05$.

(A)

\section{Serum miR-762 level}

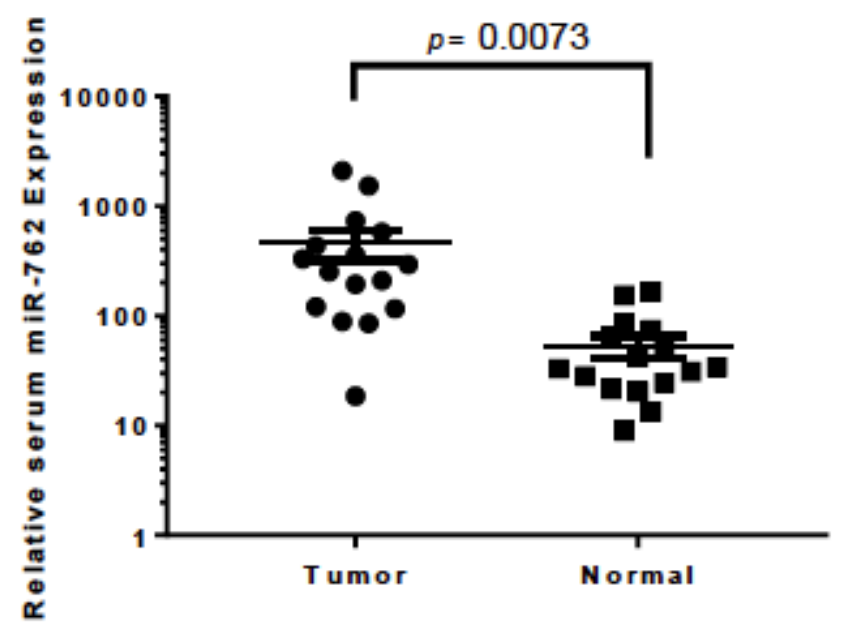

(B)

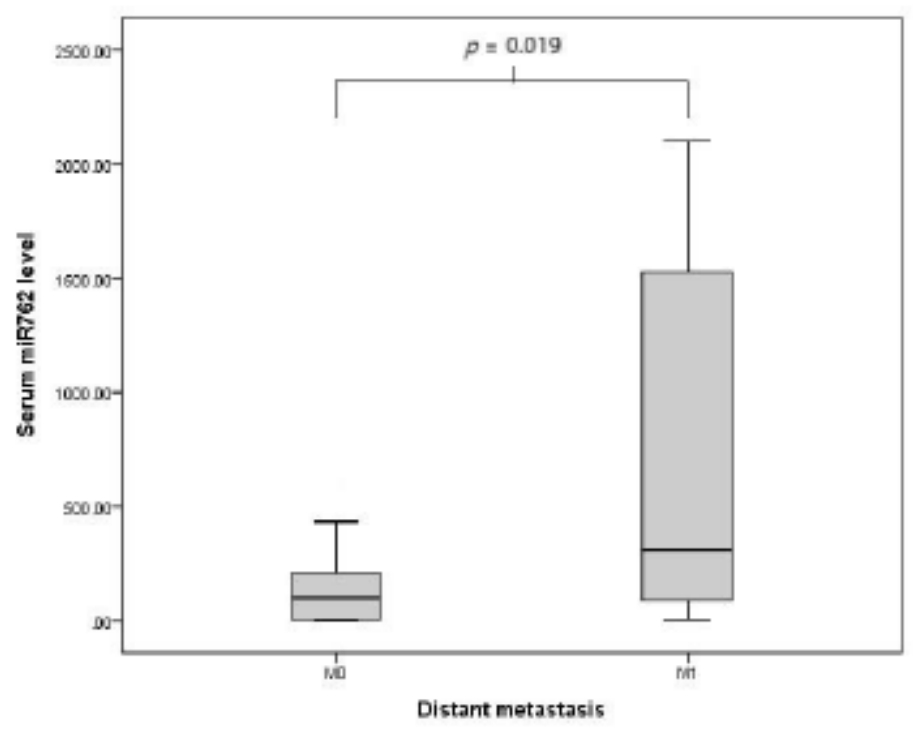

Figure 6

(A) Serum miR-762 expression in CRC patients and in control donors. Serum miR-762 expression in CRC patients $(n=20)$ was significantly higher than in control donors $(n=20),(B)$ The box plot shows the median of serum miR-762 expression, and the box shows the 25-75th percentile of serum miR-762 expression. CRC patients with distant metastasis $(M 1, n=6)$ had higher expression of serum miR-762 than $\mathrm{CRC}$ patients with no distant metastasis $(\mathrm{MO}, \mathrm{n}=14)$. The results were expressed as the mean \pm standard deviation (SD) for three independent experiments. Statistical comparisons were conducted with Student's t-test. * $\mathrm{P}<0.05$. 


\section{Supplementary Files}

This is a list of supplementary files associated with this preprint. Click to download.

- NC3RsARRIVEGuidelinesChecklistmiR762inCRC.pdf 